\title{
Synthesis of $4^{\text {th }}$ order Lossy Filters with uniform Q distribution
}

\author{
Jordi Mateu $^{1,2}$, Alberto Padilla ${ }^{1}$, Carlos Collado ${ }^{1}$, Monica Martinez-Mendoza ${ }^{3}$, Eduard Rocas ${ }^{1}$, Christoph \\ Ernst $^{3}$, Juan M. O'Callaghan ${ }^{1}$ \\ ${ }^{1}$ Universitat Politècnica de Catalunya (UPC), Campus Nord, Barcelona, Spain \\ ${ }^{2}$ Centre Tecnològic de Telecomunicacions de Catalunya (CTTC), PMT, Castelldefels, Spain \\ ${ }^{3}$ European Space Agency (ESA), ESTEC, Noordwijk ZH, The Netherlands
}

\begin{abstract}
This paper outlines the synthesis procedure for obtaining the folded coupling matrix of lossy filters of $4^{\text {th }}$ order with uniform $Q$ distribution. The method is applied to Butterworth, Chebyshev and Chebyshev with a single pair of symmetrical transmission zeros responses. Closed-form expressions to relate a prescribed insertion loss and filter bandwidth with the required resonator quality factor for an infinite $Q$ flatness filter responses have been obtained. Experimental results on a Chebyschev microstrip filter coincide with the theoretical predictions.
\end{abstract}

Index Terms - Lossy filter, insertion loss, synthesis, matrix rotation.

\section{INTRODUCTION}

Classical filter synthesis techniques require the use of high quality factor (Q) resonators to achieve filters with high selectivity and flat passband response. This might be unfeasible or impractical in space systems having stringent weight and volume restrictions. Development of synthesis techniques that take into account the limited $\mathrm{Q}$ of the filter resonators allows to optimize the filter selectivity and passband flatness, at the expense of other filter parameters (such as insertion loss) that might not be critical in channelizer filters. These techniques are usually known as lossy filter synthesis, and several works on these have been published in the recent years [1]-[9].

This paper outlines the synthesis procedure for the folded coupling network of a lossy filter and discusses issues related to insertion loss and $\mathrm{Q}$ in filters designed with such techniques. Specifically, we illustrate how to derive the required resonator $\mathrm{Q}$ for a given filter insertion loss. This is illustrated for a simple $4^{\text {th }}$ order lossy filter synthesized using a generalized technique to obtain the $(N+2) x(N+2)$ transversal coupling matrix [9] and additional circuit transformation to obtain the final network topology. This procedure allows us to obtain a uniform $\mathrm{Q}$ distribution for a given synthesized response. Note that this may be very convenient from a practical point of view, since all resonators can be implemented with the same technology, shape and dimensions, and therefore they would be subject to the same manufacturing tolerances and thermal effects.

\section{FOURTH ORDER LOSSY FILTER}

The procedure obtains the $(N+2) x(N+2)$ transversal coupling (TC) matrix from the two port admittance matrix of the filter. Then, the resulting transversal network is transformed into a folded coupling network with uniform Q distribution on the resonators and with resistive coupling between resonators. The steps and closed form formulation to obtain such uniform distribution are outlined in this section.

The synthesized response corresponds to a symmetric network where the transmission $\left(S_{21}\right)$ and reflection coefficients $\left(S_{11}\right)$ are given by $S_{21}=k \cdot S_{21}$ lossless and $S_{11}=k \cdot S_{11}$ lossless, being $\mathrm{k}<1$. Note that $\mathrm{k}$ sets the passband insertion loss as $I L(d B)=-20 \log _{10}(k)$.

\section{A. Transversal Coupling Matrix (TCM)}

This method starts from the scattering parameters of the lossy response and obtains the two port admittance matrix [Y] by means of conventional network analysis formulation [10]. Partial polynomial expansion can then be applied into [Y], obtaining:

$$
[Y]=\sum_{k=1}^{N} \frac{\left[\begin{array}{ll}
r_{11} & r_{12} \\
r_{21} & r_{22}
\end{array}\right]}{s-j\left(\lambda_{k r}+j \lambda_{k i}\right)}+\left[\begin{array}{cc}
K K & 0 \\
0 & K K
\end{array}\right]
$$

where $r_{i j}$ and $\lambda_{k}$ are the residues and roots of the polynomial expansions. In contrast with the lossless case now the roots $\lambda_{k}$ $\left(\lambda_{k}=\lambda_{k r}+j \lambda_{k i}\right)$ and the residues $r_{i j}$ are complex due to the losses in the filter. This results in the network topology of Fig.1a, where each resonator (black circles) is connected to the source $(S)$ and load $(L)$. In this topology the resonators are lossy and the couplings between the source/load and the resonators have a real and an imaginary part. From the circuit network point of view this corresponds to resistive and reactive couplings, represented by $M_{S k}$ and $M_{L k}$ in Fig. $1 \mathrm{~b}$, and with lossy resonators, defined by $B_{k}, G_{k}$ and $C_{k}$. Note that these parameters are defined from the expression (1). The partial polynomial expansion (1) results in an additional unitary diagonal matrix scaled by $K K$, which corresponds to an additionally shunt resistors $(1 / \mathrm{G})$ to the source and load [8][9].

Since the resulting transversal coupling network does not easily lend itself to a partial implementation, network transformation will be applied to obtain a more suitable network topology.

\section{B. Folded Coupling Matrix (FCM)}

Then the TCM is transformed into a FCM by means of trigonometric matrix rotations. The whole procedure to go from TCM to FCM is detailed in [10]. Doing so, we obtain a 
folded coupling topology of Fig.1c. For convenience in the subsequent distribution of losses, additional non-resonant nodes are introduced at the input and output of the filter, indicated, respectively, as $N S$ and $N L$ in Fig.1c. The resulting network can then be outlined as in Fig. 1d, and its corresponding $(N+4) x(N+4)$ coupling matrix is as follows:

\begin{tabular}{l|llllllll|}
\multicolumn{1}{c}{} & $S$ & $N S$ & 1 & 2 & 3 & 4 & $N L$ & $L$ \\
\cline { 2 - 9 }$S$ & 0 & 1 & 0 & 0 & 0 & 0 & 0 & 0 \\
$N S$ & 1 & $-j G$ & $M_{01}$ & 0 & 0 & 0 & 0 & 0 \\
1 & 0 & $M_{01}$ & $-j G_{1}$ & $M_{12}$ & 0 & $M_{14}$ & 0 & 0 \\
2 & 0 & 0 & $M_{12}$ & 0 & $M_{23}$ & 0 & 0 & 0 \\
3 & 0 & 0 & 0 & $M_{23}$ & 0 & $M_{12}$ & 0 & 0 \\
4 & 0 & 0 & $M_{14}$ & 0 & $M_{12}$ & $-j G_{1}$ & $M_{01}$ & 0 \\
$N L$ & 0 & 0 & 0 & 0 & 0 & $M_{01}$ & $-j G$ & 1 \\
$L$ & 0 & 0 & 0 & 0 & 0 & 0 & 1 & 0 \\
\cline { 2 - 10 } & & & & & & & &
\end{tabular}

where the coupling between resonators $M_{i j}$ are conventional couplings (non-resistive). The shunt resistor connected to the input and output non-resonant nodes $(G)$ is due to the imposed insertion losses $k$, and can be related to them through: $G=(1-$ $k) /(1+k)$.

The matrix above reveals that losses are not distributed throughout the network [7] [6], and the central resonators 2 and 3 result to be lossless. Moreover the central part of the matrix (i.e., the matrix elements indicated in grey), is identical to the one obtained in a lossless case. The losses in the first and last resonator $G_{l}$, can be obtained as $G_{I}=G \cdot M_{01}$ Lossless, where $M_{01}$ Lossless corresponds to the coupling between the source and first resonator in a lossless case.

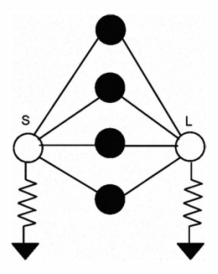

a)
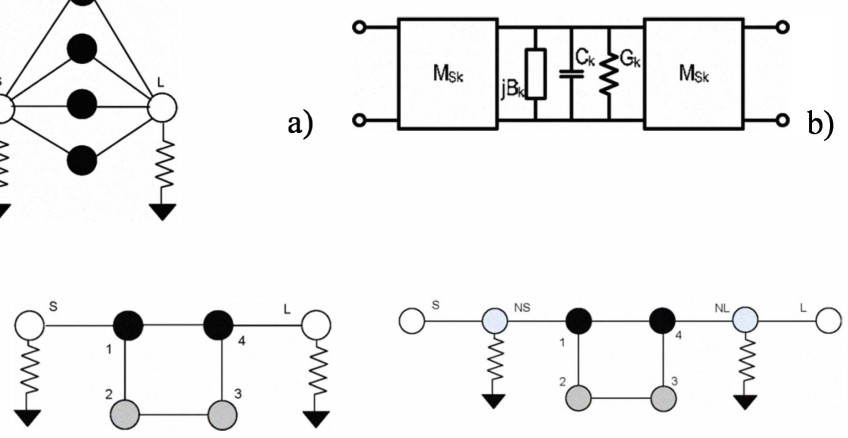

c)

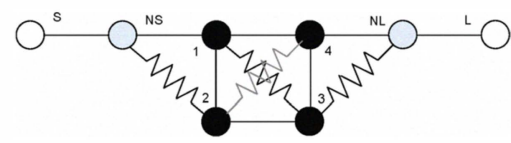

d)

e)

Fig.1.The black circles correspond to lossy resonators, the grey circles to lossless resonators, the blue circles to non-resonant nodes and the white circles correspond to the source/load. a) TC topology b) $M_{s k}$ and $M_{L k}$ represent the coupling between the source and load with each resonator, respectively. c) FC topology, d) FC topololgy with non-resonant nodes, e) topology after loss distribution.

\section{Distribution of Losses}

The next step is to shift losses to the center resonators 2 and 3 from the lossy resonators 1 and 4 . To do that, we may use hyperbolic matrix rotation [7] on the matrix above. By doing so, we are also introducing some additional resistive couplings from the non-resonant nodes to the center resonators and resistive couplings from resonator 1 to resonator 3 and from resonator 2 to resonator 4 . Details of the resistive coupling distribution are outlined in Fig.1 e.

The hyperbolic rotation is set by $c_{r}=\cosh \alpha$ and $j \cdot s_{r}=j \cdot \sinh \alpha$, where $\alpha$ is the rotation angle [7] [8].

Since the initial FCM is symmetric, we may keep the symmetry of the matrix by just applying the same hyperbolic rotation to transfer losses from 1 to 2 than that used in the transfer of loss from 4 to 3 . The following matrix shows the elements of the coupling matrix used to obtain the uniform $Q$ distribution condition.

\begin{tabular}{|c|c|c|c|c|}
\hline & $S$ & $N S$ & 1 & 2 \\
\hline$S$ & 0 & $h$ & 0 & 0 \\
\hline$N S$ & $h$ & $-j h^{2} G$ & $h c_{r} M_{0 I}$ & $j h s_{r} M_{01}$ \\
\hline 1 & 0 & $h c_{r} M_{0 l}$ & $-j\left(c_{r}^{2} G_{1}+2 s_{r} c_{r} M_{12}\right)$ & $M_{12}\left(c_{r}{ }^{2}+s_{r}{ }^{2}\right)+G_{1} c_{r} s_{r}$ \\
\hline 2 & 0 & $j h s_{r}, M_{0 l}$ & $M_{12}\left(c_{r}{ }^{2}+s_{r}{ }^{2}\right)+G_{1} c_{r} s_{r}$ & $j s_{r}\left(s_{r} G_{l}+2 c_{r} \cdot M_{12}\right)$ \\
\hline 3 & 0 & 0 & $j c_{r} s_{r}\left(M_{14}-M_{23}\right)$ & $c_{r}^{2} M_{23}-s_{r}^{2} M_{1 t}$ \\
\hline 4 & 0 & 0 & $c_{r}^{2} M_{1-1}-S_{r}^{2} M_{23}$ & $j c_{r} s_{r}\left(M_{14}-M_{23}\right)$ \\
\hline
\end{tabular}

The variable $h$ in the matrix is not yet considered at this point of the discussion, and can be assumed as $h=1$.

The lossy terms in the diagonal of the matrix (in grey) account for both the losses in the resonator (quality factor) and the resistive coupling between the resonators, whereas the lossy off-diagonal terms account for the resistive coupling between two nodes. Therefore the $\mathrm{Q}$ of each resonator can be obtained from the imaginary terms in each row.

To achieve a uniform $\mathrm{Q}$ distribution in the network one only needs to find the angle $\alpha\left(c_{r}\right.$ and $\left.s_{r}\right)$ that satisfies that the losses corresponding to the $\mathrm{Q}$ of the imaginary terms of third row (corresponding to $1^{\text {st }}$ resonator) is equal to losses corresponding to the $\mathrm{Q}$ of the imaginary terms of the fourth row (corresponding to the $2^{\text {nd }}$ resonator), as long as the resulting $\mathrm{Q}>0$.

However this does not give the minimum required $\mathrm{Q}$ for the synthesized response. To obtain the minimum $Q$ we need to move all the losses in the non-resonant nodes to the rest of the filter network. This occurs when no shunt resistance exists at the non-resonant nodes and thus, the addition of all imaginary elements in the $N S$ (or $N L$ ) row should be zero. To this end, the non-resonant node is scaled by a constant value $h$, as indicated in the matrix above.

For a minimum required $\mathrm{Q}, h$ should be:

$h=\frac{s_{r} M_{01}}{G}$

And the uniform $\mathrm{Q}$ condition results: 
$c_{r}^{2} G_{1}+2 c_{r} s_{r} M_{12}-c_{r} s_{r}\left(M_{14}-M_{23}\right)=$

$-s_{r}^{2} G_{1}-2 c_{r} s_{r} M_{12}-c_{r} s_{r}\left(M_{14}-M_{23}\right)-s_{r} h M_{01}$

By substituting (2) into (3) we obtain:

$\left(c_{r}^{2}+s_{r}^{2}\right) G_{1}+4 c_{r} s_{r} M_{12}=-s_{r}^{2} \frac{M_{01}^{2}}{G}$

Now using the values of $c_{r}$ and $s_{r}$ and applying properties of the hyperbolic functions, (4) results as:

$\left(2 G_{1}+\frac{M_{01}^{2}}{G}\right) \cosh 2 \alpha+4 M_{12} \sinh 2 \alpha-\frac{M_{01}^{2}}{G}=0$

From which we may isolate $\alpha$ as:

$\alpha=\frac{1}{2} \ln \left[\frac{\frac{M_{01}^{2}}{G} \pm \sqrt{4 G_{1} \frac{M_{01}^{2}}{G}-4 G_{1}^{2}+16 M_{12}^{2}}}{2 G_{1}+\frac{M_{01}^{2}}{G}+4 M_{12}}\right]$

The equation above has two possible solutions, only one of them potentially fulfilling the $\mathrm{Q}>0$ requirement. There are even cases where none of the two angles obtained from (6) satisfies the $\mathrm{Q}>0$ requirement. These are cases where a high selectivity is required, e.g. frequency responses with

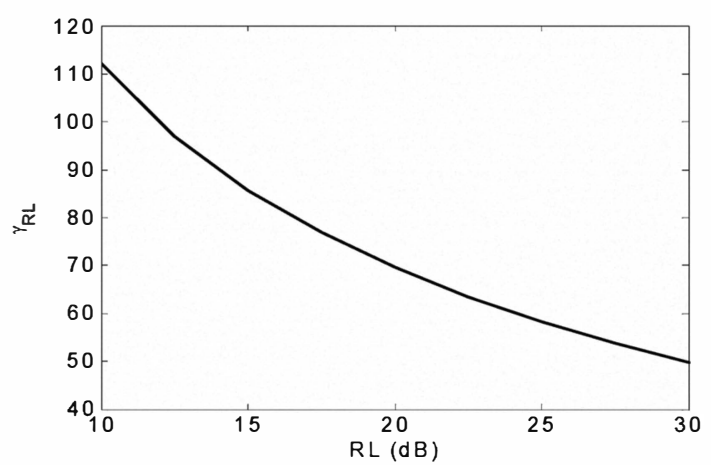

Fig. $2-\gamma_{R L}$ as a function of return losses for a fourth order Chebyshev filter.

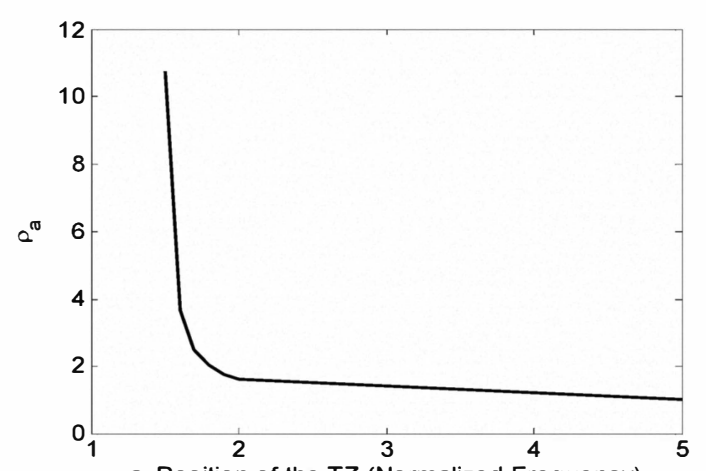

a, Position of the TZ (Normalized Frequency)

Fig. $3-\rho_{a}$ as a function of the position of the transmission zero.
TABLE I

SUMMARY OF REQUIRED Q VS. IL BUTTERWORTH

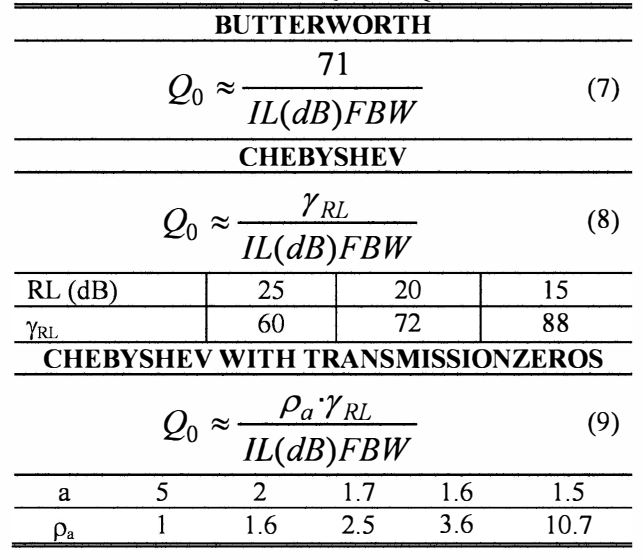

transmission zeros very close to the passband, so they would require high $\mathrm{Q}$, but at the same time the imposed insertion losses, $k$, are high, so the value of $G$ and $G_{l}$ set a given amount of losses to be distributed. In those cases uniform $\mathrm{Q}$ distribution would not be possible.

\section{REQUIRED Q VS. INSERTION LOSSES}

In this section a relation between the rotation angle $\alpha$ (6), insertion loss and minimum required $\mathrm{Q}$ is derived for:

- Butterworth Filters

- Chebychev Filters (15,20,25 dB return loss)

- Chebychev Filtres with a single pair of symmetric transmission zero at the normalized position $a$.

Table I summarizes the required $\mathrm{Q}$ for a given insertion loss. In a $4^{\text {th }}$ order Butterworth filter (7) defines a general formulation for the required Q. For a Chebyshev filter, the required $Q$ depends on the return losses by a factor $\gamma_{R L}$ as indicated in (8). Table I lists the value of $\gamma_{R L}$ for several values of RL. The dependence of $\gamma_{R L}$ on RL is depicted in Fig. 2. In this case the required $\mathrm{Q}$ increases when the $\mathrm{RL}$ decreases, which is consistent with the fact that, for a given filter order, the selectivity of the filter (slope of $\left|S_{21}\right|$ at the edge of the stop-band) increases when the RL is reduced and therefore the $\mathrm{Q}$ to achieve such performance should be higher.

When transmission zeros are introduced, the required $Q$ required is scaled by a factor $\rho_{\mathrm{a}}(9)$, whose dependence on the position of the transmission zero $(a)$ is outlined in Fig.3. The $\rho_{\mathrm{a}}$ values for several positions of the transmission zeros are also listed in Table I. As expected the required $Q$ sharply increases when the zeros are set very close to the passband, due again to the high selectivity introduced by the transmission zeros.

\section{EXAMPLE: FILTER DESIGN}

Using the procedure above we designed a microstrip Chebyshev filter with $\mathrm{RL}=20 \mathrm{~dB}$, centered at $1 \mathrm{GHz}$ with 115 $\mathrm{MHz}$ bandwidth, using a dielectric with a permittivity $\varepsilon_{\mathrm{r}}=10.2$, 
$1 \mathrm{~mm}$ thickness and a 0.003 loss tangent and with a $36 \mu \mathrm{m}$ thick cooper metallization. This allows us to achieve a $50 \Omega$ halfwave resonator with a Q of 200. By applying this Q into (9) we obtain an insertion loss of $3 \mathrm{~dB}$ which corresponds to $\mathrm{k}=0.7$.

Fig. 4 shows the synthesized (red solid line) and measured frequency response (black solid line) of the implemented filter (inset Fig.4). The resulting coupling matrix of the implemented filter is shown below. The measured frequency response exhibits flat amplitude in the passband and no rounding effects are observed in the lower band edge of the filter. The upper band edge is rounded because of the transmission zero produced by the existence of undesired cross-coupling between non-adjacent resonators. The implemented microstrip filter gives a transmission response (in the lower band edge of the passband) equivalent to a higher (1000) Q filter response.

\begin{tabular}{|llllllll|}
\hline 0 & 0.33 & 0 & 0 & 0 & 0 & 0 & 0 \\
0.33 & $-j 0.018$ & -0.34 & $j 0.018$ & 0 & 0 & 0 & 0 \\
0 & -0.34 & $-j 0.078$ & 0.906 & $j 0.037$ & 0 & 0 & 0 \\
0 & $j 0.018$ & 0.906 & $-j 0.096$ & 0.702 & $j 0.037$ & 0 & 0 \\
0 & 0 & $j 0.037$ & 0.702 & $j 0.096$ & 0.906 & $j 0.018$ & 0 \\
0 & 0 & 0 & $j 0.037$ & 0.906 & $-j 0.078$ & -0.34 & 0 \\
0 & 0 & 0 & 0 & $j 0.018$ & -0.34 & $-j 0.018$ & 0.33 \\
0 & 0 & 0 & 0 & 0 & 0 & 0.33 & 0 \\
\hline
\end{tabular}

\section{CONCLUSION}

In this work we evaluate the required $\mathrm{Q}$ for fourth order lossy filters with uniform $Q$ distribution. A closed form expression for the hyperbolic rotation angle as a function of the synthesized folded coupling matrix gives the required angle to obtain uniform distribution of Qs. The required uniform Q can then be related by general expressions with the specifications parameters of the filter: insertion losses, fractional bandwidth, return losses and position of the transmission zeros. These expressions allow filter designers to systematically account for the effects of a limited Q in the filter synthesis process. A microstrip filter with flat frequency response and equivalent to a much higher $\mathrm{Q}$ filter has been demonstrated with the method proposed.

\section{ACKNOWLEDGMENTS}

This work was supported by ESA under the contract AO 1$5477 / 07 /$ NL/GLC, by Spanish Ministry (SM) of Science and Innovation through grants TEC-2006-13248-C04-02/TCM and MAT2008-06761-C03-02; and SM of Education through a PhD fellowship for A. Padilla (AP2008-02235).

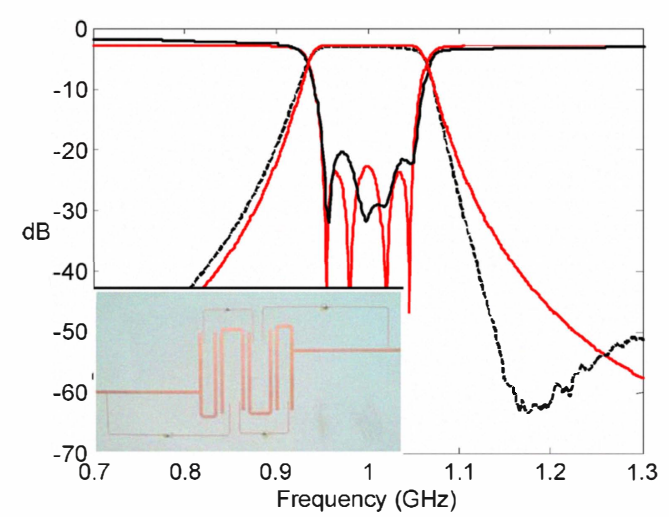

Fig. 4- Frequency response of the implemented filter. Solid red trace: simulated response with the technique detailed in this paper; black solid line: measured response. The inset shows the implemented filter.

\section{REFERENCES}

[1] J.D. Rhodes, I.C. Hunter, "Synthesis of Reflection-mode prototype networks with dissipative circuit elements" IEE Proc.Microwave Antennas Propagation, Vol. 144, No.6 December 1997.

[2] B. S. Senior, I.D. Hunter, and J.D. Rhodes, "Synthesis of lossy filters" in $32^{\text {nd }}$ Eur. Microwave Conference, Milan, Italy, 2002, pp. 401-404.

[3] I. C. Hunter, A. Guyette, and R. D. Pollard, "Passive Microwave Receive Filter Network Using Low-Q Resonators", IEEE microwave Magazine, September 2005

[4] A.C. Guyette, I. C. Hunter, R. Pollard, "Exact synthesis of Microwave Filters with nonuniform dissipation", IEEE MTT-S Digest 2007.

[5] V. Miraftab, M. Yu, "Generalized lossy microwave filter coupling matrix synthesis and design" IEEE MTT-S Digest 2008, 627-630.

[6] M.Yu, V. Miraftab, "Shrinking microwave filters" IEEE Microwave Magazine, Oct. 2008, 40-54.

[7] A.C. Guyette, I. C. Hunter, R. Pollard, "The design of microwave bandpass filters using resonators with nonuniform Q", MTT-54-11, 2006,pp. 3914-3922.

[8] J. Mateu, C. Collado, J.M O'Callaghan. Lossy filter synthesis and study of topology solution space. ESA working paper 2366. TEC-ETM/2009.142/CE

[9] V. Miraftab, Ming Yu "Advanced Coupling Matrix and Admittance Function Synthesis Techniques for Dissipative Microwave Filters" IEEE MTT. Microwave Theory and Techniques, IEEE Transactions on, vol.57, no.10, pp.2429-2438, Oct. 2009

[10] R. J. Cameron, C. M. Kudsia, R.R. Mansour, Microwave Filters for Communication systems. Fundamentals, Design, and applications, John Wiley \& Sons, 2007. 